\title{
Groups of points on abelian threefolds over finite fields
}

\author{
Yulia Kotelnikova
}

\begin{abstract}
In this paper we provide an algorithm to classify groups of points on abelian threefolds over finite fields. The classification is given in terms of the Weil polynomial of abelian varieties in a given $\mathbb{F}_{q}$-isogeny class. This work completes partial classification given in Ryb15.
\end{abstract}

\section{Introduction}

Famous results of Tsfasman Tsf85] and Xing Xin94, Xin96 concerning groups of $\mathbb{F}_{q}-$ points on elliptic curves and abelian surfaces were elegantly generalized by Rybakov in $\mathbf{R y b 1 0}$ and $\mathbf{R y b 1 2}$. This paper is devoted to classification of groups of points on abelian varieties of dimension 3. The classification was started in Ryb15. In this section we site some of Rybakov's notable theorems and give a precise problem statement.

Suppose $X$ is an abelian variety of dimension $g$ over a finite field $\mathbb{F}_{q}$ with $q=p^{r}$. Then $X\left(\mathbb{F}_{q}\right)$ is a finite abelian group. Thus there is a decomposition $X\left(\mathbb{F}_{q}\right)=\oplus_{l} X\left(\mathbb{F}_{q}\right)_{l}$ into a direct sum of its $l$-primary components.

We denote by $\mathrm{T}_{l}(X)=\lim \operatorname{ker}\left(X \stackrel{\cdot l^{m}}{\longrightarrow} X\right)\left(\overline{\mathbb{F}}_{q}\right)$ the Tate module of $X$. In case $l \neq p$ it is a $\mathbb{Z}_{l}$ module of rank $2 g$. We denote by $\operatorname{Fr}_{X}$ the Frobenius map acting on $\mathrm{T}_{l}(X)$. The characteristic polynomial $f_{X}(t)$ of $\operatorname{Fr}_{X}$ is a $q$-Weil polynomial, i. e. $f_{X}(t)$ is monic, defined over $\mathbb{Z}$ and absolute values of its roots are all equal to $\sqrt{q}$.

The following statement holds:

Proposition $1.1(\overline{\mathbf{R y b 1 0}})$. The group of points $X\left(\mathbb{F}_{q}\right)_{l}$ is isomorphic to coker $\left(1-\mathrm{Fr}_{X}\right)$.

We call the exponents of the group coker $\left(1-\operatorname{Fr}_{X}\right)$ Smith's invariants of the operator $\left(1-\operatorname{Fr}_{X}\right)$ as well as of a $\mathbb{Z}_{l}\left[1-\mathrm{Fr}_{X}\right]$-module $\mathrm{T}_{l}(X)$ (see section 2.3 for a thorough definition).

To state the results by Rybakov concerning groups of points on ordinary varieties it is convenient to introduce the notion of Newton polygon and Hodge polygon. Both of them are thought of as subsets of plane with coordinates $(x, y)$. Suppose $G$ is an l-group isomorphic to a direct sum

$$
G=\mathbb{Z} / l^{c_{1}} \oplus \mathbb{Z} / l^{c_{2}} \oplus \ldots \oplus \mathbb{Z} / l^{c_{d}}
$$

where $c_{1} \geq c_{2} \geq \ldots \geq c_{d} \geq 0$. We consider the set of points $\left(i, \sum_{j=d-i}^{d} c_{j}\right)$ and define the Hodge polygon $\operatorname{Hp}_{l}(G, d)$ of this group to be the lower boundary of its convex hull.

Let $P(t) \in \mathbb{Z}_{l}[t]$ be a monic polynomial which over $\overline{\mathbb{Q}_{l}}$ decomposes into a product

$$
P(t)=\left(t-\alpha_{1}\right)\left(t-\alpha_{2}\right) \ldots\left(t-\alpha_{d}\right),
$$

and suppose $v_{l}\left(\alpha_{1}\right) \geq v_{l}\left(\alpha_{2}\right) \ldots \geq v_{l}\left(\alpha_{d}\right) \geq 0$. We consider the set of points $\left(i, \sum_{j=d-i}^{d} v_{l}\left(\alpha_{j}\right)\right)$ and define the Newton polygon $\mathrm{Np}(P(t))$ of this polynomial to be the lower boundary of its convex hull. Alternatively, if

$$
P(t)=t^{d}+f_{1} t^{d-1}+\ldots+f_{d}
$$

then $\operatorname{Np}(P(t))$ is the lower boundary of the convex hull of the set $\left(i, v_{l}\left(f_{i}\right)\right)$.

Now we can state two beautiful theorems:

Theorem $1.2(\underline{\mathbf{R y b 1 0}})$. $\mathrm{Np}\left(f_{X}(1-t)\right)$ lies on or above $\operatorname{Hp}\left(A\left(\mathbb{F}_{q}\right)_{l}, 2 g\right)$ and their endpoints coincide.

Explicitly, if

$$
\begin{array}{ll}
A\left(\mathbb{F}_{q}\right)_{l}=\mathbb{Z} / l^{c_{1}} \oplus \mathbb{Z} / l^{c_{2}} \oplus \ldots \oplus \mathbb{Z} / l^{c_{2 g}}, & c_{1} \geq c_{2} \geq \ldots \geq c_{2 g} \geq 0 \\
f(1-t)=\left(t-\alpha_{1}\right)\left(t-\alpha_{2}\right) \ldots\left(t-\alpha_{2 g}\right), & v_{l}\left(\alpha_{1}\right) \geq v_{l}\left(\alpha_{2}\right) \ldots \geq v_{l}\left(\alpha_{2 g}\right) \geq 0,
\end{array}
$$


then the following inequalities hold

$$
\begin{aligned}
& v_{l}\left(\alpha_{1}\right) \geq c_{1} \\
& v_{l}\left(\alpha_{1}\right)+v_{l}\left(\alpha_{2}\right) \geq c_{1}+c_{2} \\
& \ldots \\
& v_{l}\left(\alpha_{1}\right)+v_{l}\left(\alpha_{2}\right)+\ldots+v_{l}\left(\alpha_{2 g}\right)=c_{1}+c_{2}+\ldots+c_{2 g} .
\end{aligned}
$$

THEOREM $1.3(\mathbf{R y b 1 0})$. Suppose $f_{X}(t)$ is separable and given an l-group $H$ such that $\operatorname{Np}\left(f_{X}(1-t)\right)$ lies on or above $\operatorname{Hp}(H, 2 g)$ and their endpoints coincide. Than there exists an abelian variety $\tilde{X}$ isogenous to $X$ such that $\tilde{X}\left(\mathbb{F}_{q}\right)_{l} \cong H$. Equivalently, suppose $f_{X}(t)$ is separable and suppose

$$
H=\mathbb{Z} / l^{c_{1}} \oplus \mathbb{Z} / l^{c_{2}} \oplus \ldots \oplus \mathbb{Z} / l^{c_{2 g}}, \quad c_{1} \geq c_{2} \geq \ldots \geq c_{2 g} \geq 0
$$

so that inequalities 1.1 hold. Than there exists an abelian variety $\tilde{X}$ isogenous to $X$ such that $\tilde{X}\left(\mathbb{F}_{q}\right)_{l} \cong H$.

The case of nonseparable $f_{X}$ (equivalently, of $X$ having noncommutative endomorphism algebra) turned up to be tricky. Tsfasman described groups of points for $\operatorname{dim} X=1$ in [Tsf85. The classification for dim $X=1,2$ and partially in case $\operatorname{dim} X=3$ was obtained by Rybakov in $\mathbf{R y b 1 0}, \mathbf{R y b 1 2}$, and $\mathbf{R y b 1 5}$. In this work we are going to apply the following result:

TheOrem 1.4 (Ryb12; Ryb15). Suppose $f_{X}(1-t)=P^{2}(t)$, $\operatorname{deg} P(t)=2$ and $P(t)$ is separable. Then the group $X\left(\mathbb{F}_{q}\right)_{l}$ is decomposable into a direct sum of two groups

$$
X\left(\mathbb{F}_{q}\right)_{l}=H_{1} \oplus H_{2}
$$

each having at most two generators such that $\mathrm{Np}(P(1-t))$ lies on or above $\mathrm{Hp}\left(H_{i}, 2\right)$ and their right borders coincide for $i=1,2$. Explicitly, suppose

$$
\begin{array}{ll}
A\left(\mathbb{F}_{q}\right)_{l}=\mathbb{Z} / l^{c_{1}} \oplus \mathbb{Z} / l^{c_{2}} \oplus \mathbb{Z} / l^{c_{3}} \oplus \mathbb{Z} / l^{c_{4}}, & c_{1} \geq \ldots \geq c_{4} \geq 0 \\
P(t)=\left(t-\alpha_{1}\right)\left(t-\alpha_{2}\right), & v_{l}\left(\alpha_{1}\right) \geq v_{l}\left(\alpha_{2}\right) \geq 0,
\end{array}
$$

then

$$
\begin{aligned}
& v_{l}\left(\alpha_{1}\right) \geq c_{1} \geq c_{2}, \\
& c_{1}+c_{4}=c_{2}+c_{3}=v_{l}\left(\alpha_{1}\right)+v_{l}\left(\alpha_{2}\right) .
\end{aligned}
$$

In the present work we complete the classification of groups of points on abelian threefolds. The following three situations are discussed:

$$
\begin{aligned}
& \text { (1) } f_{X}(t)=P^{2}(t) Q(t), \quad \operatorname{deg} P=\operatorname{deg} Q=2, \quad P(t) Q(t) \text { separable; } \\
& \text { (2) } f_{X}(t)=P(t)(t \pm \sqrt{q})^{2}, \quad \operatorname{deg} P=4, \quad P(t)(t \pm \sqrt{q}) \text { separable; } \\
& \text { (3) } f_{X}(t)=Q^{2}(t)(t \pm \sqrt{q})^{2}, \quad \operatorname{deg} Q=2, \quad Q(t)(t \pm \sqrt{q}) \text { separable. }
\end{aligned}
$$

The concept is to include the desired Tate module $\mathrm{T}_{l}(X)$ in an exact sequence of $\mathbb{Z}_{l}[T]-$ modules

$$
0 \longrightarrow \mathrm{T}_{l}(Y) \longrightarrow \mathrm{T}_{l}(X) \longrightarrow \mathrm{T}_{l}(Z) \longrightarrow 0
$$

so that both $Y$ and $Z$ have dimension at most 2. In this situation Smith's invariants of $T$ acting as one minus the Frobenius map on $\mathrm{T}_{l}(Y)$ and $\mathrm{T}_{l}(Z)$ are known by Rybakov's theorems. Due to the existence of the exact sequence, the set of Smith's invariants of $\mathrm{T}_{l}(X)$ taken together with those of $\mathrm{T}_{l}(Y)$ and $\mathrm{T}_{l}(Z)$ form a Liouville-Richardson triple (that is, the set of Smith's invariants of $\mathrm{T}_{l}(X)$ can be obtained from those of $\mathrm{T}_{l}(Y)$ and $\mathrm{T}_{l}(Z)$ by applying the Liouville-Richardson rule). Such triples appear in many problems, for instance, if one wants to find a decomposition of a $\mathfrak{g l}_{n}-$ module $V_{\lambda} \otimes V_{\mu}$ into a sum of irreducible modules. Some other applications are listed in the Section 2, Finally, Liouville-Richardson triples are exactly the ones satisfying certain set of inequalities.

The fact that Smith's invariants of modules in an exact triple form a Liouville-Richardson triple was discovered J. A Green, T. Klein; the proof can be found in Mac95. In the papers [Tho85 and SQS99 divisibility conditions on the Smith's invariants are obtained from the theorem by Green and Klein. These results are surveyed in Ful00, especially Section 2. The idea to apply these results to the problem of finding groups of points on abelian varieties was introduced in Mei15 but both main theorems of this paper were incorrect.

We describe this set of inequalities in Section 2 following W. Fulton's survey Ful00. In 2.1 we recall theorems on eigenavalues of sums of Hermitian matrices by A. Knutson and T. Tao in [KT99. The aim of Section 2.3 is to state the results concerning Smith's invariants using the definitions and theorems of Section 2.1 as well as examples given in Section 2.2. In Section 2.3 we also shorten the list of inequalities thus we obtain Corollary 2.16 from Theorem 2.9.

In Section 3 we explain thoroughly in what way Corollary 2.16 is applied to the problem of computation of groups of points on threefolds with Weil polynomials from the list 1.2 In Sections 3.1 and 3.2 we conduct the computations and obtain the lists of inequalities. In Section 4 the results are summarized in three theorems concerning each Weil polynomial from the list 1.2 . 


\section{On Horn conjecture}

Relation between sets of Smith's invariants (invariant factors) of a pair of matrices over a (local principal ideal) domain $R$ and one of their product is described by the conditions appearing also in Horn conjecture. Interpretation in terms of Hermitian matrices provides some tools for dealing with these conditions which turns up to be helpful when it comes to Smith's invariants (see Example 2.6 and Lemma 2.11). In the first half of this section we cite celebrated results on sums of Hermitian matrices, and the second half is devoted to Smith's invariants.

2.1. Sums of Hermitian matrices. The first part of this section is dedicated to manipulations with hermitian matrices. We follow the text Ful00 in which the results of [KT99 are surveyed.

Recall that a hermitian operator is diagonalizable, all its eigenvalues are real and eigenspaces corresponding to distinct eigenvalues are orthogonal to each other. Suppose $(A, B, C=A+B)$ is a triple of hermitian $n \times n$ matrices acting on a vector space $V$ with eigenvalues $\mathbf{a}=\left(a_{1} \geq a_{2} \geq \ldots \geq a_{n}\right), \mathbf{b}=\left(b_{1} \geq b_{2} \geq \ldots \geq b_{n}\right), \mathbf{c}=\left(c_{1} \geq c_{2} \geq \ldots \geq c_{n}\right)$ respectively. It turns up that there is a list of inequalities of the form

$\left(*_{I J K}\right)$

$$
\sum_{i \in I} a_{i}+\sum_{j \in J} b_{j} \geq \sum_{k \in K} c_{k}
$$

with $I, J, K$ certain subsets of $\{1,2, \ldots, n\}$ giving necessary and sufficient conditions for a triple of hermitian matrices $A$, $B, C=A+B$ with eigenvalues $\mathbf{a}, \mathbf{b}, \mathbf{c}$ to exist. We shall now describe the triples $(I, J, K)$ such that the corresponding inequalities $\left(*_{I J K}\right)$ belong to the list.

For this purpose we introduce some more notation. A subscript always denotes number of elements in the set, whereas superscripts may denote various additional information on elements of the set such as upper or lower bounds. In case an expression is taken in parentheses, the superscript denotes the degree.

$$
\begin{aligned}
& M_{n}=\{1,2, \ldots, n\} ; \\
& \Lambda \leq n=\left\{\left(i_{1}<i_{2}<\ldots<i_{p}\right) \in\left(\mathbb{Z}_{\geq 0}\right)^{p} \quad \mid \quad 1 \leq i_{1} ; \quad i_{p} \leq n\right\} ; \\
& \left.U_{p}^{n}=\left\{\begin{array}{ll}
(I, J, K) \in(\Lambda \leq n \\
p
\end{array}\right)^{3} \quad \mid \quad \sum_{i \in I} i+\sum_{j \in J} j=\sum_{k \in K} k+\frac{p(p+1)}{2}\right\} .
\end{aligned}
$$

Desired triples are organized in sets $T_{p}^{n} \subset U_{p}^{n}$. First, we set $T_{1}^{n}=U_{1}^{n}$. The sets $T_{p}^{n}$ are now defined recursively by

$$
\begin{array}{r}
T_{p}^{n}=\left\{(I, J, K) \in U_{p}^{n} \quad \mid \forall 1 \leq r<p \quad \forall(F, G, H) \in T_{r}^{p}\right. \\
\left.\sum_{f \in F} i_{f}+\sum_{g \in G} j_{g} \geq \sum_{h \in H} k_{h}+\frac{r(r+1)}{2}\right\} .
\end{array}
$$

We also introduce sets

$$
\begin{aligned}
& L_{n}^{\mathbb{R}}=\left\{\left(\lambda_{1} \geq \lambda_{2} \geq \ldots \geq \lambda_{n}\right)\right\} \subset(\mathbb{R})^{n} \\
& L_{n}^{+}=\left\{\left(\lambda_{1} \geq \lambda_{2} \geq \ldots \geq \lambda_{n}\right) \mid \lambda_{n} \geq 0\right\} \subset\left(\mathbb{Z}_{\geq 0}\right)^{n} .
\end{aligned}
$$

Now we can state three main theorems concerning eigenvalues of sums of Hermitian matrices.

Theorem 2.1 (Ful00, Theorem 1). Let $(\mathbf{a}, \mathbf{b}, \mathbf{c})$ be a triple in $\left(L_{n}^{\mathbb{R}}\right)^{3}$. There exist a triple $A, B, C=A+B$ of hermitian $n \times n$ matrices with eigenvalues $\mathbf{a}, \mathbf{b}$, and $\mathbf{c}$ respectively if and only if

$$
\sum_{\mathbf{a}} a+\sum_{\mathbf{b}} b=\sum_{\mathbf{c}} c
$$

and $\left(*_{I J K}\right)$ holds for all $(I, J, K) \in T_{p}^{n}$ for each $p$.

Theorem 2.2 (Ful00, Theorem 2). Let $A, B, C=A+B$ be a triple of hermitian $n \times n$ matrices with eigenvalues $(\mathbf{a}, \mathbf{b}, \mathbf{c}) \in\left(L_{n}^{\mathbb{R}}\right)^{3}$ and let

$$
\sum_{i \in I} a_{i}+\sum_{j \in J} b_{j}=\sum_{k \in K} c_{k}
$$

for some triple $(I, J, K) \in T_{p}^{n}$. Then there is a p-dimensional subspace $W \subset V$ invariant under the action of $A, B$ and $C$.

Due to orthogonality of eigenspaces this in fact implies that the matrices $A, B$ and $C$ have a couple of complementary common invariant subspaces in $V$.

We define a map $\lambda: \underset{p}{\leq} \rightarrow L_{p}^{+}$as follows:

$$
\lambda(I)=\left(i_{p}-p \geq i_{p-1}-p+1 \geq \ldots \geq i_{1}-1\right) .
$$

Theorem 2.3 ([Ful00], Theorem 5). Let $(I, J, K)$ be a triple in $U_{p}^{n}$. There exist a triple $A, B, C=A+B$ of hermitian matrices with eigenvalues $\lambda(I), \lambda(J)$, and $\lambda(K)$ respectively if and only if $(I, J, K) \in T_{n}^{p}$.

Hence if $(I, J, K) \in T_{p}^{n}$ one may consider some triple of hermitian matrices with eigenvalues $\lambda(I), \lambda(J)$, and $\lambda(K)$. We shall (abusively) write $A(I), B(J)$, and $C(K)$ meaning that we pick up any triple, although there might exist many of them. For example, consider a triple $(I, J, K) \in T_{p}^{n}$ with $J=(1,2, \ldots, p)$. In this case we say that $B(J)=0$ and $A(I)=C(K)$, even though the latter ones are not specified. 
REMARK 2.4. It turns up that the inequalities $\left(*_{I J K}\right)$ for all $1 \leq p \leq n-1$ and all $(I, J, K) \in T_{p}^{n}$ together with

$$
\begin{aligned}
& a_{1} \geq a_{2} \geq \ldots \geq a_{n} \\
& b_{1} \geq b_{2} \geq \ldots \geq b_{n} \\
& c_{1} \geq c_{2} \geq \ldots \geq c_{n} \\
& \sum_{a \in \mathbf{a}} a+\sum_{b \in \mathbf{b}}=\sum_{c \in \mathbf{c}}
\end{aligned}
$$

are not independent for $n \geq 6$. In particular, for $n=6$ the only redundant inequality is

$$
a_{1}+a_{3}+a_{5}+b_{1}+b_{3}+b_{5} \geq c_{2}+c_{4}+c_{5}
$$

This inequality follows from 2.1. However, in practice, Lemma 2.13 and Corollary 2.11 leaves this particular inequality out of consideration. See also [Ful00, Example 1.

2.2. Examples. We give some examples of triples in $T_{p}^{n}$.

EXAMPLE 2.5. By definition, $T_{1}^{n}$ consists of triples

$$
(\{i\},\{j\},\{k=i+j-1\}) .
$$

It is not hard to describe explicitly the set $T_{2}^{n}$. A triple $(I, J, K) \in U_{2}^{n}$ belongs to $T_{2}^{n}$ if and only if

$$
\begin{aligned}
& i_{1}+j_{1} \leq k_{1}+1 \\
& i_{2}+j_{1} \leq k_{2}+1 \\
& i_{1}+j_{2} \leq k_{2}+1
\end{aligned}
$$

See also enlightening Hor62] theorem 8 and also theorem 9 for the explicit description of $T_{3}^{n}$.

Example 2.6. Complementary inequalities. One can look for complementary inequalities of the form

$$
\sum_{i \in I^{c}} a_{i}+\sum_{j \in J^{c}} b_{j} \leq \sum_{k \in K^{c}} c_{k}
$$

by subtracting $\left(*_{I J K}\right)$ from the equality

$$
\sum_{\mathbf{a}} a+\sum_{\mathbf{b}} b=\sum_{\mathbf{c}} c
$$

For example, the set $T_{n-1}^{n}$ consists of triples

$$
\left(M_{n} \backslash\{i\}, M_{n} \backslash\{j\}, M_{n} \backslash\{i+j-n\}\right)
$$

which give the inequalities

$$
a_{i}+b_{j} \leq c_{i+j-n} .
$$

Suppose $(I, J, K) \in T_{p}^{n}$. One obtains a complementary triple from matrices $n-A(I), n-B(J), n-C(K)$.

ExAmPLE 2.7. Lidskii inequalities. Let $J=\{1,2, \ldots, p\}$. The inequalities $i_{\alpha}+j_{1} \leq k_{\alpha}+1$ for all $\alpha$ combined with

$$
\sum_{i \in I} i+\sum_{j \in J} j=\sum_{k \in K} k+\frac{p(p+1)}{2}
$$

imply that $I=K$. Thus we have

$$
\sum_{i \in I} a_{i}+\sum_{j=1}^{p} b_{j}=\sum_{i \in I} c_{i}
$$

As discussed above, in this situation $B(J)=0$ and $A(I)=C(K)$.

ExAmPLE 2.8. The previous example can be easily improved in a following way. Suppose $J=\{s+1, s+2, \ldots, s+p\}$. Again the inequalities $i_{\alpha}+j_{1} \leq k_{\alpha}+1$ for all $\alpha$ combined with

$$
\sum_{i \in I} i+\sum_{j \in J} j=\sum_{k \in K} k+\frac{p(p+1)}{2}
$$

imply that $i_{\alpha}=k_{\alpha}+s$ for all $\alpha$. In this case $B(J)$ is a scalar matrix. 
2.3. Smith's invariants. For our purposes we will need the versions of these statements with Smith's invariants. For simplicity let $R=\mathbb{Z}_{l}$ and let $\mathcal{A} \in \operatorname{Mat}_{s \times s}(R)$. Then $R^{s} / \mathcal{A} R^{s}$ is a finite abelian $l$-group, hence it has the form

$$
R^{s} / \mathcal{A} R^{s}=\mathbb{Z} / l^{a_{1}} \mathbb{Z} \oplus \mathbb{Z} / l^{a_{2}} \mathbb{Z} \oplus \ldots \oplus \mathbb{Z} / l^{a_{s}} \mathbb{Z}
$$

with $a_{1} \geq a_{2} \geq \ldots \geq a_{s} \geq 0$. The set $\mathfrak{a}=\left\{a_{1} \geq a_{2} \geq \ldots \geq a_{s}\right\}$ is said to be Smith's invariants of the matrix $\mathcal{A}$, as well as of the group $R^{s} / \mathcal{A} R^{s}$.

Suppose there is an exact sequence of $R[T]$-modules

$$
0 \longrightarrow R^{s} \longrightarrow R^{s+t} \longrightarrow R^{t} \longrightarrow 0
$$

with the action of $T$ on $R^{s}, R^{t}$, and $R^{s+t}$ defined by matrices $\mathcal{A}, \mathcal{B}$, and $\mathcal{C}$ respectively. In this case $\mathcal{C}$ is of the form

$$
\mathcal{C}=\left(\begin{array}{ll}
\mathcal{A} & \mathcal{X} \\
0 & \mathcal{B}
\end{array}\right)
$$

for some $\mathcal{X} \in \operatorname{Mat}_{t \times s}(R)$. Moreover, from

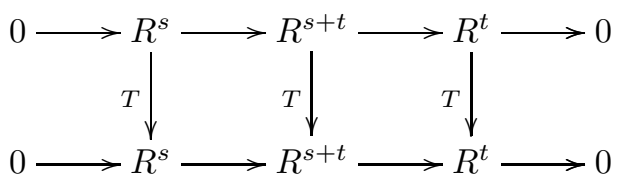

by the snake lemma we obtain

$$
0 \longrightarrow R^{s} / \mathcal{A} R^{s} \longrightarrow R^{s+t} / \mathcal{C} R^{s+t} \longrightarrow R^{t} / \mathcal{B} R^{t} \longrightarrow 0 .
$$

We shall now state the theorem which relates invariant factors of certain triples of matrices.

Theorem 2.9 (Ful00], Theorem 10; SQS99], Corollary 3.2). Let $(\mathfrak{a}, \mathfrak{b}, \mathfrak{c})$ be a triple in $L_{s}^{+} \times L_{t}^{+} \times L_{s+t}^{+}$. Then there exist such matrices $\mathcal{A} \in \operatorname{Mat}_{s \times s}(R), \mathcal{B} \in \operatorname{Mat}_{t \times t}(R), \mathcal{X} \in \operatorname{Mat}_{t \times s}(R)$ and

$$
\mathcal{C}=\left(\begin{array}{ll}
\mathcal{A} & \mathcal{X} \\
0 & \mathcal{B}
\end{array}\right)
$$

such that $\mathfrak{a}, \mathfrak{b}, \mathfrak{c}$ are Smith's invariants of $\mathcal{A}, \mathcal{B}, \mathcal{C}$ respectively if and only if

$$
\sum_{a \in \mathfrak{a}} a+\sum_{b \in \mathfrak{b}} b=\sum_{c \in \mathfrak{c}} c
$$

and for all $p$ and all $(I, J, K) \in T_{p}^{n}$

$$
\sum_{i \in I \cap M_{s}} a_{i}+\sum_{j \in J \cap M_{t}} b_{j} \geq \sum_{k \in K} c_{k} .
$$

REMARK 2.10. One can easily extend the above theorem to the case of arbitrary principal ideal domain.

The list 2.2 of inequalities to check contains redundant ones. For example, conditions

$$
\sum_{i \in I \cap M_{s}} a_{i}+\sum_{j \in J \cap M_{t}} b_{j} \geq \sum_{k \in K} c_{k}
$$

for $I=J=K=\{1,2, \ldots, p\}$ for $p>s, t$ follow from

$$
\sum_{a \in \mathfrak{a}} a+\sum_{b \in \mathfrak{b}} b=\sum_{c \in \mathfrak{c}} c .
$$

The lemmas below imply that list of inequalities of the form 2.2 can in fact be significantly shortened.

Lemma 2.11. Suppose $(I, J, K) \in T_{p}^{n}$ and there is an $\alpha$ such that $i_{\alpha-1}+1<i_{\alpha}$ (or $\alpha=1$ and $1<i_{1}$ ). Then there exists $\beta$ such that

(1) $k_{\beta-1}+1<k_{\beta}\left(\right.$ or $\beta=1$ and $\left.1<k_{\beta}\right)$;

(2) $\left(I^{\prime}, J, K^{\prime}\right) \in T_{p}^{n}$ with $I^{\prime}=I \backslash\left\{i_{\alpha}\right\} \sqcup\left\{i_{\alpha}-1\right\}$, and $K^{\prime}=K \backslash\left\{k_{\beta}\right\} \cup\left\{k_{\beta}-1\right\}$.

First, we give a restatement of the lemma:

Lemma 2.11. Suppose $A, B, C=A+B$ are $p \times p$ matrices with integer nonnegative eigenvalues and $\mathbf{a}, \mathbf{b}$, and $\mathbf{c}$ respectively and suppose $a_{\alpha} \neq 0$ for some $\alpha \in M_{p}$. Then there exist

(立) $\beta \in M_{p}$ such that $c_{\beta} \neq 0$

$(\tilde{2})$ a triple of matrices $A^{\prime}, B^{\prime}, C^{\prime}=A^{\prime}+B^{\prime}$ with eigenvalues $\mathbf{a} \backslash\left\{a_{\alpha}\right\} \sqcup\left\{a_{\alpha}-1\right\}$, $\mathbf{b}$, and $\mathbf{c} \backslash\left\{c_{\beta}\right\} \cup\left\{c_{\beta}-1\right\}$ respectively. 
Proof of Lemmas 2.11 and 2.11]. Theorem 2.3 implies that Lemma 2.11 follows from Lemma 2.11 applied to $(A, B, C)=(A(I), B(J), C(K))$. Thus it suffices to prove the later one.

Suppose there is no $\beta$ for which ( $\tilde{1})$ holds. Then $C=0$ and therefore $A=B=0$ which contradicts the definition of $\alpha$.

We prove the lemmas by induction in $p$. If $p=1$, then the statement holds.

Let matrices $(A, B, C)$ have dimensions $p>1$. Recall that by Theorem 2.1 a triple $(A, B, C)$ satisfies certain list of inequalities $\left(*_{F G H}\right)$. If there is no inequality $\left(*_{F G H}\right)$ occurring with equality then for any $\beta$ satisfying $(\tilde{1})$ the sets $\mathbf{a} \backslash\left\{a_{\alpha}\right\} \sqcup\left\{a_{\alpha}-1\right\}, \mathbf{b}$, and $\mathbf{c} \backslash\left\{c_{\beta}\right\} \cup\left\{c_{\beta}-1\right\}$ still satisfy conditions of Theorem 2.1. Therefore there exists a desired triple of matrices $\left(A^{\prime}, B^{\prime}, C^{\prime}\right)$.

Otherwise the matrices $A, B, C$ have a pair of common invariant complementary proper subspaces $W$ and $W^{\perp}$. Let the set of eigenvalues of $\left.A\right|_{W}$ contain $a_{\alpha}$. Then the induction step is applicable to the triple $\left(\left.A\right|_{W},\left.B\right|_{W},\left.C\right|_{W}\right)$, thus there exists a triple which we denote by $\left(\left(\left.A\right|_{W}\right)^{\prime},\left(\left.B\right|_{W}\right)^{\prime},\left(\left.C\right|_{W}\right)^{\prime}\right)$. Then the desired triple of matrices is $\left(\left.\left(\left.A\right|_{W}\right)^{\prime} \oplus A\right|_{W^{\perp}},\left(\left.B\right|_{W}\right)^{\prime} \oplus\right.$ $\left.\left.B\right|_{W^{\perp}},\left.\left(\left.C\right|_{W}\right)^{\prime} \oplus C\right|_{W^{\perp}}\right)$.

Definition 2.12. Suppose $(I, J, K) \in T_{p}^{s+t}$ and set

$$
\begin{aligned}
& \tilde{I}=I \cap M_{s}, \\
& \tilde{J}=J \cap M_{t} .
\end{aligned}
$$

We say that $(I, J, K) \in \tilde{T}_{p}^{s, t}$ if

$$
\begin{aligned}
& I \backslash \tilde{I}=\{s+1, s+2, \ldots s+\alpha\}, \\
& J \backslash \tilde{J}=\{t+1, t+2, \ldots t+\beta\}
\end{aligned}
$$

for some $\alpha$ and $\beta$.

Corollary 2.13. Theorem 2.9 is true with $T_{p}^{s+t}$ replaced by $\tilde{T}_{p}^{s, t}$.

Lemma 2.14. Suppose $(I, J, K) \in \tilde{T}_{p}^{s, t}$ are as in definition 2.12, Then

$$
\# \tilde{I}+\# \tilde{J} \geq p .
$$

Moreover, suppose

Then there exists $\gamma \notin K$ such that

$$
\# \tilde{I}+\# \tilde{J}>p
$$

$$
(I \sqcup\{s+\alpha+1\}, J \sqcup\{t+\beta+1\}, K \sqcup\{\gamma\}) \in T_{p+1}^{s+t} .
$$

Proof. In case

$$
\# \tilde{I}+\# \tilde{J}<p,
$$

the triple $(\{\# \tilde{I}+1\},\{\# \tilde{J}+1\},\{\# \tilde{I}+\# \tilde{J}+1\})$ belongs to $T_{1}^{p}$. Therefore,

$$
i_{\# \tilde{I}+1}+j_{\# \tilde{J}+1} \leq k_{\# \tilde{I}+\# \tilde{J}+1}+1
$$

should be satisfied. However,

$$
k_{\# \tilde{I}+\# \tilde{J}+1}+1 \leq s+t+1<s+t+2=i_{\# \tilde{I}+1}+j_{\# \tilde{J}+1}
$$

Now suppose

$$
\# \tilde{I}+\# \tilde{J}>p
$$

By the trace equality we obtain $\gamma=s+t+\alpha+\beta-p+1$. Then

$$
\# \tilde{I}+\# \tilde{J}=p-\alpha+p-\beta>p,
$$

implies that $\gamma \leq s+t$. Moreover, by Example 2.6.

$$
k_{p} \leq i_{p}+j_{p}-p=s+t+\alpha+\beta-p=\gamma-1,
$$

and thus, $\gamma \notin K$. Finally, matrices $A(I) \oplus(s+\alpha-p), B(J) \oplus(t+\beta-p), C(K) \oplus(s+t+\alpha+\beta-2 p)$ satisfy Theorem 2.3. hence

$$
(I \sqcup\{s+\alpha+1\}, J \sqcup\{t+\beta+1\}, K \sqcup\{\gamma\}) \in T_{p+1}^{s+t} .
$$

Definition 2.15. We say that $(I, J, K) \in T_{p}^{s, t}$ if and only if $(I, J, K) \in \tilde{T}_{p}^{s, t}$ and

$$
\# \tilde{I}+\# \tilde{J}=p .
$$

Corollary 2.16. Theorem 2.9 is true with $T_{p}^{s+t}$ replaced by $T_{p}^{s, t}$. 
Proof. By Corollary 2.13 we replace $T_{p}^{s+t}$ by $\tilde{T}_{p}^{s, t}$. We want to show that there is no essential inequalities lying in $\tilde{T}_{p}^{s, t} \backslash T_{p}^{s, t}$. Consider a triple $(I, J, K) \in \tilde{T}_{p}^{s, t}$ such that \# $\tilde{I}+\# \tilde{J}>p$. Then by Theorem 2.9 this triple corresponds to the inequality

$$
\sum_{\tilde{I}} a_{i}+\sum_{\tilde{J}} b_{j} \geq \sum_{k \in K} c_{k}
$$

This inequality is redundant since by Lemma 2.14 there is a triple $(I \sqcup\{s+\alpha+1\}, J \sqcup\{t+\beta+1\}, K \sqcup\{\gamma\}) \in T_{p+1}^{s+t}$ which corresponds to a stronger inequality

$$
\sum_{\tilde{I}} a_{i}+\sum_{\tilde{J}} b_{j} \geq \sum_{k \in K} c_{k}+c_{\gamma}
$$

\section{On groups of points}

Recall that we intend to study abelian threefolds with characteristic polynomials of the form

(1.2)

(1) $f_{X}(t)=P^{2}(t) Q(t), \quad \operatorname{deg} P=\operatorname{deg} Q=2, \quad P(t) Q(t)$ separable;

(2) $f_{X}(t)=P(t)(t \pm \sqrt{q})^{2}, \quad \operatorname{deg} P=4, \quad P(t)(t \pm \sqrt{q})$ separable;

(3) $f_{X}(t)=Q^{2}(t)(t \pm \sqrt{q})^{2}, \quad \operatorname{deg} Q=2, \quad Q(t)(t \pm \sqrt{q})$ separable.

The following general method is used. Suppose $f_{X}(t)$ is decomposable over $\mathbb{Z}$ into a product of coprime factors $f_{X}(t)=P(t) Q(t)$, the variety $X$ can be included in an exact sequence of abelian varieties (as fppf sheaves)

$$
0 \longrightarrow Y \longrightarrow X \longrightarrow Z \longrightarrow 0
$$

with $Y$ and $Z$ lying in isogeny classes corresponding to polynomials $P$ and $Q$. Thus there is also an exact sequence of Tate modules, and even of $\mathbb{Z}_{l}[T]$-modules

$$
0 \longrightarrow \mathrm{T}_{l}(Y) \longrightarrow \mathrm{T}_{l}(X) \longrightarrow \mathrm{T}_{l}(Z) \longrightarrow 0,
$$

with $T$ acting as $\operatorname{Fr}_{X}, \operatorname{Fr}_{Y}$, and $\operatorname{Fr}_{Z}$ on $X, Y$, and $Z$ respectively.

Thus if one fixes $Y$ and $Z$ and in particular, $\mathbb{Z}_{l}[T]$-modules $\mathrm{T}_{l}(Y)$ and $\mathrm{T}_{l}(Z)$, then by the results of Section 2.3 Smith's invariants of the module $\mathrm{T}_{l}(X)$ should satisfy the conditions of Theorem 2.9

Conversely, let $Y$ and $Z$ be abelian varieties with coprime Weil polynomials $P$ and $Q$ and suppose there is an exact sequence of $\mathbb{Z}_{l}[T]$-modules

$$
0 \longrightarrow \mathrm{T}_{l}(Y) \longrightarrow \mathcal{T}_{l} \longrightarrow \mathrm{T}_{l}(Z) \longrightarrow 0,
$$

with $T$ acting as $1-\operatorname{Fr}_{Y}$ on $\mathrm{T}_{l}(Y)$ and as $1-\operatorname{Fr}_{Z}$ on $\mathrm{T}_{l}(Z)$. Since $P$ and $Q$ are coprime, $T$ acts semisimply on $\mathcal{T}_{l}$. Therefore one might construct an inclusion $\mathcal{T}_{l} \subset \mathrm{T}_{l}(Y \times Z)$ as a Frobenius invariant submodule. Then there exists an abelian variety $X$ which is $l$-isogenuous to $(Y \times Z)$ such that $\mathcal{T}_{l} \cong \mathrm{T}_{l}(X)$ as $\mathbb{Z}_{l}[T]$-modules (see for example, $\mathbf{R y b 1 0}$ ). Thus given $Y$ and $Z$ (in particular, $\mathbb{Z}_{l}[T]$-modules $\mathrm{T}_{l}(Y)$ and $\mathrm{T}_{l}(Z)$ ), and given a module $\mathcal{T}_{l}$ satisfying conditions of Theorem 2.9 or in fact Corollary 2.16, there exists an abelian variety $X$ such that $\mathcal{T}_{l} \cong \mathrm{T}_{l}(X)$.

Therefore to list possible exponents of groups of points on abelian threefolds we should consider all possible pairs $Y$ and $Z$ and list varieties $X$ whose Smith's invariants satisfy Corollary 2.16.

Since both $Y$ and $Z$ have dimensions at most 2 we recall part of the classification of Tate modules of abelian varieties of small dimensions is given in Ryb10, $\mathbf{R y b 1 2}$, and Ryb15. Consider a variety $Y$ with $\operatorname{dim} Y \leq 2$ and suppose $f_{X}(1-t)$ is the characteristic polynomial of the operator $1-F r_{Y}$. If $f_{X}$ is separable, then by Theorems 1.2 and 1.3 groups of points of abelian varieties isgenuous to $X$ are exactly the groups $G$ whose Hodge polygon $\operatorname{Hp}(H, 2 g)$ lies on or below $\operatorname{Np}\left(f_{X}(1-t)\right)$. Otherwise $f_{X}$ is a square and this case is treated by Theorem 1.4 .

First, we intend to give an alternative proof of a stronger version of Theorem 1.4

Theorem 3.1 ([Ryb15 $)$. Let $f(t)$ be a Weil polynomial, $f(1-t)=P^{r} Q^{s}$ where $Q$ divides $P, \operatorname{deg} P=2$ and $\operatorname{deg} Q=1$ and $P$ is separable. Then $G$ is the group of points on some abelian variety with Weil polynomial $f$ if and only if

$$
G \cong \oplus_{j=1}^{r} G_{j} \oplus H
$$

where $G_{j}$ are l-primary abelian groups such that $\mathrm{Np}_{l}(P(t))$ lies on or above $\operatorname{Hp}\left(G_{j}, 2\right)$ for all $1 \leq j \leq r$, and $H \cong$ $\left(\mathbb{Z}_{l} / Q(0) \mathbb{Z}_{l}\right)^{s}$.

Proof. First, the case $f(t)=(t \pm \sqrt{q})^{s}$ is trivial.

Otherwise, the statement is an easy consequence of the classification of finite torsionfree modules over orders with cyclic index presented below. Let $\mathfrak{A}$ be a separable commutative algebra of finite rank $n$ over $\mathbb{Q}_{l}$, suppose $\mathcal{O}_{\mathfrak{A}} \subset \mathfrak{A}$ is a maximal order in $\mathfrak{A}$, and suppose $\mathcal{O} \subset \mathcal{O}_{\mathfrak{A}} \subset \mathfrak{A}$ is an order with cyclic index that is $\mathcal{O}_{\mathfrak{A}} / \mathcal{O}$ is a cyclic group.

By a lattice in $\mathfrak{A}$ we mean an additive subgroup $M \subset \mathfrak{A}$ which is a free $\mathbb{Z}_{l}$-module of rank $n$. For a lattice $M \subset \mathfrak{A}$ the set $\mathcal{O}=\{\xi \in \mathcal{A} \mid \xi M \subset M\}$ is an order and we say that the lattice $M$ belongs to the order $\mathcal{O}$. 
TheOREM $3.2([\mathbf{B F 6 5}])$. Suppose $\mathcal{O} \subset \mathfrak{A}$ is an order with cyclic index in a separable commutative finite $\mathbb{Q}_{l}$-algebra $\mathfrak{A}$ and let $M$ be a finitely generated torsionfree $\mathcal{O}$-module. Then there exists the chain of ideals of $\mathfrak{A}$

$$
\mathfrak{A} \supseteq \mathcal{B}_{1} \supset \mathcal{B}_{2} \supset \ldots \supset \mathcal{B}_{s}
$$

such that in each algebra $\mathcal{B}_{i}$ there exists a lattice $B_{i}$ which belongs to a $\mathcal{B}_{i}$-order with cyclic index, and there exists an $\mathcal{O}$-decomposition

$$
M \cong B_{1} \oplus B_{2} \oplus \ldots \oplus B_{s-1} \oplus B_{s} .
$$

TheOREm 3.3 ([BF65]). Suppose $\mathcal{O} \subset \mathfrak{A}$ is an order with cyclic index in a separable commutative finite $\mathbb{Q}_{l}$-algebra $\mathfrak{A}$ and let $M \subset \mathfrak{A}^{s}$ be a module of rank $s$ over $\mathcal{O}$. Then there exists the chain of orders of $\mathfrak{A}$

$$
\mathcal{O} \subseteq \mathcal{O}_{1} \subset \mathcal{O}_{2} \subset \ldots \subset \mathcal{O}_{s} \subseteq \mathcal{O}_{\mathfrak{A}}
$$

and the decomposition

$$
M \cong \mathcal{O}_{1} \oplus \mathcal{O}_{2} \oplus \ldots \oplus \mathcal{O}_{s-1} \oplus \mathcal{M}_{s}
$$

where $\mathcal{M}_{s} \subset \mathfrak{A}$ is a lattice which belongs to $\mathcal{O}_{s}$. Equivalently, there is a decomposition

$$
M \cong M_{1} \oplus M_{2} \oplus \ldots \oplus M_{s}
$$

where $M_{i} \subset \mathfrak{A}$ are lattices which belong to $\mathcal{O}_{i}$.

Recall that the Frobenius morphism acts separably on the Tate module $\mathrm{T}_{l}(X)$. Therefore $\mathrm{T}_{l}(X)$ is a module over an order $\mathbb{Z}_{l}\left[1-\mathrm{Fr}_{X}\right]$ in an algebra $\mathbb{Q}_{l}[T] / P(T)$, which is separable over $\mathbb{Q}_{l}$. The algebra $\mathbb{Q}_{l}[T] / P(T)$ is isomorphic either to a quadratic extension $K$ of $\mathbb{Q}_{l}$ or to $\mathbb{Q}_{l} \times \mathbb{Q}_{l}$. In both cases all orders $\mathcal{O} \subset \mathbb{Q}_{l}[T] / P(T)$ are with cyclic index.

Therefore $\mathrm{T}_{l}(X)$ is a $\mathbb{Z}_{l}\left[1-\operatorname{Fr}_{X}\right]$-module as in Theorem 3.2. There is a decomposition into a sum of two $\mathbb{Z}_{l}\left[1-\operatorname{Fr}_{X}\right]-$ modules

$$
\mathrm{T}_{l}(X) \cong B_{1} \oplus B_{2}
$$

and to each $B_{i}$ Theorem 3.3 applies. Thus

$$
\begin{gathered}
\mathrm{T}_{l}(X) \cong M_{1} \oplus M_{2} \oplus \ldots \oplus M_{r} \oplus \mathbb{Z}_{l}^{s} . \\
\operatorname{coker}\left(\mathrm{T}_{l}(X) \stackrel{1-\operatorname{Fr}_{X}}{\longrightarrow} \mathrm{T}_{l}(X)\right) \cong \oplus_{i=1}^{r} \operatorname{coker}\left(M_{i} \stackrel{1-\operatorname{Fr}_{X}}{\longrightarrow} M_{i}\right) \oplus \operatorname{coker}\left(\mathbb{Z}_{l}^{s} \stackrel{1-\operatorname{Fr}_{X}}{\longrightarrow} \mathbb{Z}_{l}^{s}\right) .
\end{gathered}
$$

The operator $\left(1-\operatorname{Fr}_{X}\right)$ acts on the modules $M_{i}$ with characteristic polynomial equal to $P(t)$. Both polynomials are separable, thus Theorems 1.2 and 1.3 apply. Therefore a group $G_{i}$ is equal to

$$
\operatorname{coker}\left(M_{i} \stackrel{1-\operatorname{Fr}_{X}}{\longrightarrow} M_{i}\right)
$$

for some lattice $M_{i}$ if and only if their Hodge polygons $\operatorname{Hp}\left(G_{i}, 2\right)$ lie on or below Newton polygon $\operatorname{Np}(P(t))$.

The operator $\left(1-\operatorname{Fr}_{X}\right)$ acts on the modules $\mathbb{Z}_{l}^{s}$ with characteristic polynomial equal to $Q(t)$, that is $\left(1-\operatorname{Fr}_{X}\right)$ is multiplication by $Q(0)$. Therefore

$$
H \cong \operatorname{coker}\left(\mathbb{Z}_{l}^{s} \stackrel{1-\mathrm{Fr}_{X}}{\longrightarrow} \mathbb{Z}_{l}^{s}\right) \cong\left(\mathbb{Z}_{l} / Q(0) \mathbb{Z}_{l}\right)^{s}
$$

as desired.

3.1. Let $f_{X}(t)=P^{2}(t) Q(t)$ with $\operatorname{deg} P=\operatorname{deg} Q=2, P Q$ separable. Than there is an exact sequence

$$
0 \longrightarrow Y \longrightarrow X \longrightarrow Z \longrightarrow 0
$$

where $Y$ and $Z$ are associated with polynomials $P^{2}$ and $Q$ respectively. Suppose groups $Y\left(\mathbb{F}_{q}\right)_{l}$ and $Z\left(\mathbb{F}_{q}\right)_{l}$ have exponents $\mathfrak{a}=\left(a_{1}, a_{2}, a_{3}, a_{4}\right)$ and $\mathfrak{b}=\left(b_{1}, b_{2}\right)$ respectively. Suppose $\mathfrak{c}=\left(c_{1}, \ldots, c_{6}\right)$ are exponents of $X\left(\mathbb{F}_{q}\right)_{l}$. Sets $\mathfrak{a}$ and $\mathfrak{b}$ depend on $P$ and $Q$ and satisfy the conditions of Theorems 1.2 and 1.4. We shall list the inequalities which by Theorem 2.9 relate $\mathfrak{c}$ to $\mathfrak{a}$ and $\mathfrak{b}$.

By Corollary 2.16 the essential inequalities are encoded in triples $(I, J, K) \in T_{p}^{4,2}$. There are only 4 possibilities for $\tilde{J}=J \cap M_{2}$, namely $\emptyset,\{1\},\{2\},\{1,2\}$. We use complementary inequalities instead of inequalities of size at least 4 .

The inequalities with $p=1$ and $p=5$ are listed separately due to their importance.

1: Inequalities of size 1 and 5. Those are

$$
\begin{array}{lrrr}
{[0]} & (I=\{i\}, J=\{1\}) & a_{i}+b_{1} \geq c_{i}, & 1 \leq i \leq 4 \\
{[0]} & (I=\{i\}, J=\{2\}) & a_{i}+b_{2} \geq c_{i+1}, & 1 \leq i \leq 4 \\
{[1]} & (I=\{5\}, J=\{1\}) & b_{1} \geq c_{5} & \\
{[2]} & (I=\{5\}, J=\{2\}) & b_{2} \geq c_{6} & \\
{[3]} & (I=\{i\}, J=\{3\}) & a_{i} \geq c_{i+2}, & 1 \leq i \leq 4 \\
{[4]} & \left(I=M_{6} \backslash\{6\}, J=M_{6} \backslash\{1\}\right) & b_{1} \leq c_{1} & \\
{[5]} & \left(I=M_{6} \backslash\{6\}, J=M_{6} \backslash\{2\}\right) & b_{2} \leq c_{2} & \\
{[6]} & \left(I=M_{6} \backslash\{i\}, J=M_{6} \backslash\{6\}\right) & a_{i} \leq c_{i}, & 1 \leq i \leq 4 .
\end{array}
$$



form

2: The set $\tilde{J}=\{1,2\}$ corresponds to Lidskii inequalities, see Example 2.7 The complementary inequalities have the

and follow from 3.26$]$.

$$
\sum_{i \in I} a_{i} \leq \sum_{i \in I} c_{i}
$$

By Example 2.8, the inequalities with $\tilde{J}=\emptyset$ have the form

$$
\sum_{i \in I} a_{i} \geq \sum_{i \in I} c_{i+2}
$$

and follow from $3.2[3]$.

3: Let $\tilde{J}=\{2\}$. Then by Example 2.8, we have $k_{\alpha}=i_{\alpha}+1$ for every $\alpha$. The corresponding inequalities are

$$
\text { [7] } \quad I \subset M_{4} \quad \sum_{i \in I} a_{i}+b_{2} \geq \sum_{i \in I} c_{i+1}+c_{6}
$$

4: Let $\tilde{J}=\{1\}$, that is $J=\{1,3,4, \ldots, p+1\}$. Then for all $1 \leq \alpha \leq p$ we have

$$
\begin{aligned}
& i_{\alpha}+j_{p} \geq k_{\alpha}+p, \\
& i_{\alpha}+j_{1} \leq k_{\alpha}+1,
\end{aligned}
$$

and therefore $i_{\alpha} \leq k_{\alpha} \leq i_{\alpha}+1$. By

$$
\sum_{I} i+\sum_{J} j=\sum_{K} k
$$

there must be only one $\beta$ such that $i_{\beta}=k_{\beta}$ and $i_{\alpha}+1=k_{\alpha}$ for $\alpha \neq \beta$. If these conditions are satisfied then we may set

$$
\begin{aligned}
& A(I)=\operatorname{diag}\left(i_{1}-1, \ldots i_{p}-p\right), \\
& B(J)=\operatorname{diag}(1, \ldots, 1,0,1, \ldots, 1), \\
& C(K)=\operatorname{diag}\left(k_{1}-1, \ldots k_{p}-p\right),
\end{aligned}
$$

with $B(J)_{\beta, \beta}=0$. These matrices satisfy Theorem 2.3 therefore $(I, J, K) \in T_{p}^{n}$.

Since we only need triples $(I, J, K) \in T_{p}^{s, t}$, it suffices to consider sets $I$ containing 5 and not containing 6 . Indeed, $\# I \backslash M_{4}=p-\# I \cap M_{4}=\# J \cap M_{2}=1$. The corresponding inequalities of size 2 and 4 are

$$
\begin{array}{rrrr}
{[8]} & (I=\{i, 5\}, K=\{i, 6\}) & a_{i}+b_{1} \geq c_{i}+c_{6}, & 1 \leq i \leq 4 \\
{[9]} & (I=\{i, 5\}, K=\{i+1,5\}) & a_{i}+b_{1} \geq c_{i+1}+c_{5}, & 1 \leq i \leq 3 \\
{[10]} & \left(I=M_{5} \backslash\{i\}, K=M_{6} \backslash\{1,(i+2)\}\right) & a_{i}+b_{2} \leq c_{1}+c_{i+2}, & 1 \leq i \leq 4 \\
{[11]} & \left(I=M_{5} \backslash\{i\}, K=M_{6} \backslash\{2,(i+1)\}\right) & a_{i}+b_{2} \leq c_{2}+c_{i+1}, & 1 \leq i \leq 3 .
\end{array}
$$

Note that 3.3 7] for $\# I=1$ and 3.4 8 ] imply [3.2[0], as predicted by Corollary 2.16

The inequalities of size 3 are

$$
\begin{array}{llll}
{[12]} & (I=\{1,2,5\}, K=\{1,3,6\}) & a_{1}+a_{2}+b_{1} \geq c_{1}+c_{3}+c_{6} \\
{[13]} & (I=\{1,2,5\}, K=\{2,3,5\}) & a_{1}+a_{2}+b_{1} \geq c_{2}+c_{3}+c_{5} \\
{[14]} & (I=\{1,3,5\}, K=\{1,4,6\}) & a_{1}+a_{3}+b_{1} \geq c_{1}+c_{4}+c_{6} \\
{[14]} & (I=\{1,3,5\}, K=\{2,3,6\}) & a_{1}+a_{3}+b_{1} \geq c_{2}+c_{3}+c_{6} \\
{[15]} & (I=\{1,3,5\}, K=\{2,4,5\}) & a_{1}+a_{3}+b_{1} \geq c_{2}+c_{4}+c_{5} \\
{[16]} & (I=\{1,4,5\}, K=\{1,5,6\}) & a_{1}+a_{4}+b_{1} \geq c_{1}+c_{5}+c_{6} \\
{[17]} & (I=\{1,4,5\}, K=\{2,4,6\}) & a_{1}+a_{4}+b_{1} \geq c_{2}+c_{4}+c_{6} \\
{[18]} & (I=\{2,3,5\}, K=\{2,4,6\}) & a_{2}+a_{3}+b_{1} \geq c_{2}+c_{4}+c_{6} \\
{[19]} & (I=\{2,3,5\}, K=\{3,4,5\}) & a_{2}+a_{3}+b_{1} \geq c_{3}+c_{4}+c_{5} \\
{[20]} & (I=\{2,4,5\}, K=\{2,5,6\}) & a_{2}+a_{4}+b_{1} \geq c_{2}+c_{5}+c_{6} \\
{[21]} & (I=\{2,4,5\}, K=\{3,4,5\}) & a_{2}+a_{4}+b_{1} \geq c_{3}+c_{4}+c_{5} \\
{[22]} & (I=\{3,4,5\}, K=\{3,5,6\}) & a_{3}+a_{4}+b_{1} \geq c_{3}+c_{5}+c_{6}
\end{array}
$$

Inequalities 3.5[14] are equivalent due to Theorem 1.4 .

3.2. Let $f_{X}(t)=P(t)(t \pm \sqrt{q})^{2}, P(t)$ separable and $P(\mp \sqrt{q}) \neq 0$ or $P(t)=Q^{2}(t)$ with $Q(t)$ separable and $Q(\mp \sqrt{q}) \neq$ 0 . Than there is an exact sequence

$$
0 \longrightarrow Y \longrightarrow X \longrightarrow Z \longrightarrow 0
$$

where $Y$ and $Z$ are associated with polynomials $P(t)$ and $(t \pm \sqrt{q})^{2}$ respectively. Note that $1-\mathrm{Fr}_{Z}$ acts as multiplication by $b=v_{l}(1 \pm q)$, so its Smith's invariants are $\mathfrak{b}=(b, b)$. Suppose $Y\left(\mathbb{F}_{q}\right)_{l}$ and $X\left(\mathbb{F}_{q}\right)_{l}$ have exponents $\mathfrak{a}=\left(a_{1}, a_{2}, a_{3}, a_{4}\right)$ and $\mathfrak{c}=\left(c_{1}, \ldots, c_{6}\right)$ respectively. Sets $\mathfrak{a}$ and $\mathfrak{b}$ satisfy Theorem 1.2. Moreover, in case $P=Q^{2}$ Theorem 1.4 is applicable, and therefore $a_{1}+a_{4}=a_{2}+a_{3}$. Again we list inequalities relating $\mathfrak{c}$ to $\mathfrak{a}$ and $\mathfrak{b}$.

To list the inequalities we only consider triples $(I, J, K) \in T_{p}^{4,2}$ with $\tilde{J}$ one of $\emptyset,\{1\},\{2\},\{1,2\}$. Since $\mathfrak{b}=(b, b)$, by Lemma 2.11 we don't need to consider sets $J$ containing 2 but not 1 . Thus the case $\tilde{J}=\{2\}$ is now redundant. 
1: Inequalities of size 1 and 5. Those are

$$
\begin{array}{lrrll}
{[0]} & (I=\{i\}, J=\{1\}) & a_{i}+b & \geq c_{i}, & 1 \leq i \leq 4 \\
{[1]} & (I=\{5\}, J=\{1\}) & b & \geq c_{5} & \\
{[2]} & (I=\{i\}, J=\{3\}) & a_{i} \geq c_{i+2}, & 1 \leq i \leq 4 \\
{[3]} & \left(I=M_{6} \backslash\{6\}, J=M_{6} \backslash\{2\}\right) & b & \leq c_{2} & \\
{[4]} & \left(I=M_{6} \backslash\{i\}, J=M_{6} \backslash\{6\}\right) & a_{i} \leq c_{i}, & 1 \leq i \leq 4 .
\end{array}
$$

2: For $\tilde{J}=\{1,2\}$ the complementary inequalities have the form

$$
\sum_{i \in I} a_{i} \leq \sum_{i \in I} c_{i}
$$

and follow from 3.6 4] (see Example 2.7).

By Example 2.8, the inequalities with $\tilde{J}=\emptyset$ have the form

$$
\sum_{i \in I} a_{i} \geq \sum_{i \in I} c_{i+2}
$$

and follow from 3.62$]$.

3: The case $\tilde{J}=\{1\}$ is treated in 3.1 part 4. In this situation $I$ contains 5 and does not contain 6 . Moreover, $i_{\beta}=k_{\beta}$ for some $1 \leq \beta \leq 4$ and $i_{\alpha}+1=k_{\alpha}$ for $\alpha \neq \beta$. The corresponding inequalities of sizes 2 and 4 are

$$
\begin{array}{lrrrr}
{[5]} & (I=\{i, 5\}, K=\{i, 6\}) & a_{i}+b \geq c_{i}+c_{6}, & 1 \leq i \leq 4 \\
{[6]} & (I=\{i, 5\}, K=\{i+1,5\}) & a_{i}+b \geq c_{i+1}+c_{5}, & 1 \leq i \leq 3 \\
{[7]} & \left(I=M_{5} \backslash\{i\}, K=M_{6} \backslash\{1,(i+2)\}\right) & a_{i}+b \leq c_{1}+c_{i+2}, & 1 \leq i \leq 4 \\
{[8]} & \left(I=M_{5} \backslash\{i\}, K=M_{6} \backslash\{2,(i+1)\}\right) & a_{i}+b \leq c_{2}+c_{i+1}, & 1 \leq i \leq 3 .
\end{array}
$$

Note that 3.7 5$]$ imply [3.6[0].

The inequalities of size 3 are

$$
\begin{array}{rlll}
{[9]} & (I=\{1,2,5\}, K=\{1,3,6\}) & a_{1}+a_{2}+b \geq c_{1}+c_{3}+c_{6} \\
{[10]} & (I=\{1,2,5\}, K=\{2,3,5\}) & a_{1}+a_{2}+b \geq c_{2}+c_{3}+c_{5} \\
{[11]} & (I=\{1,3,5\}, K=\{1,4,6\}) & a_{1}+a_{3}+b \geq c_{1}+c_{4}+c_{6} \\
{[12]} & (I=\{1,3,5\}, K=\{2,3,6\}) & a_{1}+a_{3}+b \geq c_{2}+c_{3}+c_{6} \\
{[13]} & (I=\{1,3,5\}, K=\{2,4,5\}) & a_{1}+a_{3}+b \geq c_{2}+c_{4}+c_{5} \\
{[14]} & (I=\{1,4,5\}, K=\{1,5,6\}) & a_{1}+a_{4}+b \geq c_{1}+c_{5}+c_{6} \\
{[15]} & (I=\{1,4,5\}, K=\{2,4,6\}) & a_{1}+a_{4}+b \geq c_{2}+c_{4}+c_{6} \\
{[16]} & (I=\{2,3,5\}, K=\{2,4,6\}) & a_{2}+a_{3}+b \geq c_{2}+c_{4}+c_{6} \\
{[17]} & (I=\{2,3,5\}, K=\{3,4,5\}) & a_{2}+a_{3}+b \geq c_{3}+c_{4}+c_{5} \\
{[18]} & (I=\{2,4,5\}, K=\{2,5,6\}) & a_{2}+a_{4}+b \geq c_{2}+c_{5}+c_{6} \\
{[19]} & (I=\{2,4,5\}, K=\{3,4,5\}) & a_{2}+a_{4}+b \geq c_{3}+c_{4}+c_{5} \\
{[20]} & (I=\{3,4,5\}, K=\{3,5,6\}) & a_{3}+a_{4}+b \geq c_{3}+c_{5}+c_{6}
\end{array}
$$

In case $P=Q^{2}$ inequalities 3.8[15] and 3.8[16] are identical. 


\section{Main result}

Computation conducted in the previous section combined with Theorems 1.2 and 1.4 imply the following three results.

Theorem 4.1. Suppose $X / \mathbb{F}_{q}$ is an abelian threefold with characteristic polynomial of Frobenius map equal to $f_{X}(t)=$ $P^{2}(t) Q(t)$ with $\operatorname{deg} P=\operatorname{deg} Q=2, P Q$ separable, suppose $\left(m_{1} \geq m_{2}\right)$ and $\left(n_{1} \geq n_{2}\right)$ are absolute values in $\mathbb{Z}_{l}$ of zeros of $P(1-t)$ and $Q(1-t)$ respectively. Then there exists an isogenous variety $\tilde{X}$ with group of points

$$
\tilde{X}\left(\mathbb{F}_{q}\right)_{l}=\mathbb{Z} / l^{c_{1}} \oplus \mathbb{Z} / l^{c_{2}} \oplus \ldots \oplus \mathbb{Z} / l^{c_{6}}
$$

if and only if there exist sets of numbers $\mathfrak{a}=\left(a_{1}, a_{2}, a_{3}, a_{4}\right), \mathfrak{b}=\left(b_{1}, b_{2}\right)$ such that

(1) $c_{1}+\ldots+c_{6}=2 m_{1}+2 m_{2}+n_{1}+n_{2}$

(2) $a_{2} \leq a_{1} \leq m_{1}$

$a_{1}+a_{4}=a_{2}+a_{3}=m_{1}+m_{2}$

$n_{2} \leq b_{2} \leq b_{1} \leq n_{1}$

$b_{1}+b_{2}=n_{1}+n_{2}$

(3) the following inequalities hold

$\begin{array}{lrll}{[1]} & b_{1} \geq c_{5} & \\ {[2]} & b_{2} \geq c_{6} & \\ {[3]} & a_{i} \geq c_{i+2}, & \\ {[4]} & b_{1} \leq c_{1} & \\ {[5]} & b_{2} \leq c_{2} & 1 \leq i \leq 4 \\ {[6]} & a_{i} \leq c_{i}, & I \subset M_{4} \\ {[7]} & \sum_{i \in I} a_{i}+b_{2} \geq \sum_{i \in I} c_{i+1}+c_{6} & 1 \leq i \leq 4 \\ {[8]} & a_{i}+b_{1} \geq c_{i}+c_{6}, & 1 \leq i \leq 3 \\ {[9]} & a_{i}+b_{1} \geq c_{i+1}+c_{5}, & 1 \leq i \leq 4 \\ {[10]} & a_{i}+b_{2} \leq c_{1}+c_{i+2}, & 1 \leq i \leq 3 \\ {[11]} & a_{i}+b_{2} \leq c_{2}+c_{i+1}, & \\ {[12]} & a_{1}+a_{2}+b_{1} \geq c_{1}+c_{3}+c_{6} & \\ {[13]} & a_{1}+a_{2}+b_{1} \geq c_{2}+c_{3}+c_{5} & \\ {[14]} & a_{1}+a_{3}+b_{1} \geq c_{2}+c_{3}+c_{6} & \\ {[15]} & a_{1}+a_{3}+b_{1} \geq c_{2}+c_{4}+c_{5} & \\ {[16]} & a_{1}+a_{4}+b_{1} \geq c_{1}+c_{5}+c_{6} & \\ {[17]} & a_{1}+a_{4}+b_{1} \geq c_{2}+c_{4}+c_{6} & \\ {[18]} & a_{2}+a_{3}+b_{1} \geq c_{2}+c_{4}+c_{6} & \\ {[19]} & a_{2}+a_{3}+b_{1} \geq c_{3}+c_{4}+c_{5} & \\ {[20]} & a_{2}+a_{4}+b_{1} \geq c_{2}+c_{5}+c_{6} & \\ {[21]} & a_{2}+a_{4}+b_{1} \geq c_{3}+c_{4}+c_{5} & \\ {[22]} & a_{3}+a_{4}+b_{1} \geq c_{3}+c_{5}+c_{6} & \end{array}$


Theorem 4.2. Suppose $X / \mathbb{F}_{q}$ is an abelian threefold with characteristic polynomial of Frobenius map equal to $f_{X}(t)=$ $P(t)(t \pm \sqrt{q})^{2}$ with $P(t) \cdot(t \pm \sqrt{q})$ separable, suppose $\left(m_{1} \geq m_{2} \geq m_{3} \geq m_{4}\right)$ are absolute values in $\mathbb{Z}_{l}$ of zeros of $P(1-t)$ and $v_{l}(1 \pm \sqrt{q})=b$. Then there exists an isogenous variety $\tilde{X}$ with group of points

$$
\tilde{X}\left(\mathbb{F}_{q}\right)_{l}=\mathbb{Z} / l^{c_{1}} \oplus \mathbb{Z} / l^{c_{2}} \oplus \ldots \oplus \mathbb{Z} / l^{c_{6}}
$$

if and only if there exists a set of numbers $\mathfrak{a}=\left(a_{1}, a_{2}, a_{3}, a_{4}\right)$ such that

(1) $c_{1}+\ldots+c_{6}=m_{1}+\ldots+m_{4}+2 b$

(2) $a_{1} \leq m_{1}$

$$
\begin{aligned}
& a_{1}+a_{2} \leq m_{1}+m_{2} \\
& a_{1}+a_{2}+a_{3} \leq m_{1}+m_{2}+m_{3} \\
& a_{1}+a_{2}+a_{3}+a_{4}=m_{1}+m_{2}+m_{3}+m_{4}
\end{aligned}
$$

(3) the following inequalities hold

$\begin{array}{lrll}{[1]} & b & \geq c_{5} & \\ {[2]} & a_{i} & \geq c_{i+2}, & 1 \leq i \leq 4 \\ {[3]} & b & \leq c_{2} & \\ {[4]} & a_{i} & \leq c_{i}, & 1 \leq i \leq 4 \\ {[5]} & a_{i}+b & \geq c_{i}+c_{6}, & 1 \leq i \leq 4 \\ {[6]} & a_{i}+b & \geq c_{i+1}+c_{5}, & 1 \leq i \leq 3 \\ {[7]} & a_{i}+b & \leq c_{1}+c_{i+2}, & 1 \leq i \leq 4 \\ {[8]} & a_{i}+b & \leq c_{2}+c_{i+1}, & 1 \leq i \leq 3 \\ {[9]} & a_{1}+a_{2}+b \geq c_{1}+c_{3}+c_{6} & \\ {[10]} & a_{1}+a_{2}+b \geq c_{2}+c_{3}+c_{5} & \\ {[11]} & a_{1}+a_{3}+b & \geq c_{1}+c_{4}+c_{6} & \\ {[12]} & a_{1}+a_{3}+b \geq c_{2}+c_{3}+c_{6} & \\ {[13]} & a_{1}+a_{3}+b & \geq c_{2}+c_{4}+c_{5} & \\ {[14]} & a_{1}+a_{4}+b & \geq c_{1}+c_{5}+c_{6} & \\ {[15]} & a_{1}+a_{4}+b \geq c_{2}+c_{4}+c_{6} & \\ {[16]} & a_{2}+a_{3}+b & \geq c_{2}+c_{4}+c_{6} & \\ {[17]} & a_{2}+a_{3}+b & \geq c_{3}+c_{4}+c_{5} & \\ {[18]} & a_{2}+a_{4}+b & \geq c_{2}+c_{5}+c_{6} & \\ {[19]} & a_{2}+a_{4}+b & \geq c_{3}+c_{4}+c_{5} & \\ {[20]} & a_{3}+a_{4}+b & \geq c_{3}+c_{5}+c_{6} & \\ & & & \end{array}$


TheOREm 4.3. Suppose $X / \mathbb{F}_{q}$ is an abelian threefold with characteristic polynomial of Frobenius map equal to $f_{X}(t)=$ $P^{2}(t)(t \pm \sqrt{q})^{2}, P(t) \cdot(t \pm \sqrt{q})$ separable, suppose $\left(m_{1} \geq m_{2}\right)$ are absolute values in $\mathbb{Z}_{l}$ of zeros of $P(1-t)$ and $v_{l}(1 \pm \sqrt{q})=b$. Then there exists an isogenous variety $\tilde{X}$ with group of points

$$
\tilde{X}\left(\mathbb{F}_{q}\right)_{l}=\mathbb{Z} / l^{c_{1}} \oplus \mathbb{Z} / l^{c_{2}} \oplus \ldots \oplus \mathbb{Z} / l^{c_{6}}
$$

if and only if there exists a set of numbers $\mathfrak{a}=\left(a_{1}, a_{2}, a_{3}, a_{4}\right)$ such that

(1) $c_{1}+\ldots+c_{6}=2 m_{1}+2 m_{2}+2 b$

(2) $a_{2} \leq a_{1} \leq m_{1}$

$$
a_{1}+a_{4}=a_{2}+a_{3}=m_{1}+m_{2}
$$

(3) the following inequalities hold

$$
\begin{array}{rrll}
{[1]} & b & \geq c_{5} & \\
{[2]} & a_{i} & \geq c_{i+2}, & 1 \leq i \leq 4 \\
{[3]} & b & \leq c_{2} & \\
{[4]} & a_{i} & \leq c_{i}, & 1 \leq i \leq 4 \\
{[5]} & a_{i}+b & \geq c_{i}+c_{6}, & 1 \leq i \leq 4 \\
{[6]} & a_{i}+b & \geq c_{i+1}+c_{5}, & 1 \leq i \leq 3 \\
{[7]} & a_{i}+b & \leq c_{1}+c_{i+2}, & 1 \leq i \leq 4 \\
{[8]} & a_{i}+b & \leq c_{2}+c_{i+1}, & 1 \leq i \leq 3 \\
{[9]} & a_{1}+a_{2}+b \geq c_{1}+c_{3}+c_{6} & \\
{[10]} & a_{1}+a_{2}+b \geq c_{2}+c_{3}+c_{5} & \\
{[11]} & a_{1}+a_{3}+b \geq c_{2}+c_{3}+c_{6} & \\
{[12]} & a_{1}+a_{3}+b \geq c_{2}+c_{4}+c_{5} & \\
{[13]} & a_{1}+a_{4}+b \geq c_{1}+c_{5}+c_{6} & \\
{[14]} & a_{1}+a_{4}+b & \geq c_{2}+c_{4}+c_{6} & \\
{[15],[16]} & a_{2}+a_{3}+b & \geq c_{2}+c_{4}+c_{6} & \\
{[17]} & a_{2}+a_{3}+b & \geq c_{3}+c_{4}+c_{5} & \\
{[18]} & a_{2}+a_{4}+b & \geq c_{2}+c_{5}+c_{6} & \\
{[19]} & a_{2}+a_{4}+b & \geq c_{3}+c_{4}+c_{5} & \\
{[20]} & a_{3}+a_{4}+b & \geq c_{3}+c_{5}+c_{6} &
\end{array}
$$

\section{References}

[BF65] Z. I. Borevich and D. K. Faddeev, Representations of orders with a cyclic index, Trudy Mat. Inst. Steklou. 80 (1965), 51-65 (Russian); English transl., Proc. Steklou Inst. Math. 80 (1965), 56-72.

[Ful00] W. Fulton, Eigenvalues, invariant factors, highest weights, and Schubert calculus, Bull. Amer. Math. Soc. 37 (2000), 209-249, available at https://arxiv.org/pdf/math/9908012.pdf

[Hor62] A. Horn, Eigenvalues of sums of Hermitian matrices, Pacic J. Math. 12 (1962), 225-241.

[KT99] A. Knutson and T. Tao, The honeycomb model of $G L_{n}(\mathbb{C})$ tensor products I: proof of the saturation conjecture, J. Amer. Math. Soc. 12 (1999), 1055-1090.

[Mac95] I. G. Macdonald, Symmetric Functions and Hall Polynomials, 2nd ed., Oxford Mathematical Monographs, Oxford University Press, USA, 1995

[Mei15] P. Meisner, Groups structure of abelian varieties (2015), available at https://arxiv.org/pdf/1503.04326v1.pdf

[Ryb10] S. Rybakov, The groups of points on abelian varieties over finite fields, Cent. Eur. J. Math. 8(2) (2010), 282-288, available at https://arxiv.org/pdf/0903.0106.pdf

[Ryb12] S. Rybakov, The groups of points on abelian surfaces over finite fields, Arithmetic, Geometry, Cryptography and Coding Theory, Cont. Math. $\mathbf{5 7 4}$ (2012), 151-158, available at https://arxiv.org/pdf/1007.0115.pdf

[Ryb15] S. Rybakov, On classification of groups of points on abelian varieties over finite fields (2015), available at https://arxiv.org/pdf/1401.1652.pdf

[SQS99] A. P. Santana, J. F. Queiró, and E. Marques de Sá, Group representations and matrix spectral problems, Linear and Multilinear Algebra 46 (1999), 1-23.

[Tho85] R. Thompson, Smith invariants of a product of integral matrices, Contemp. Math 47 (1985), 401-435.

[Tsf85] M. Tsfasman, The group of points of an elliptic curve over a finite field, Theory of numbers and its applications (1985), $286-287$.

[Xin94] Ch. Xing, The structure of the rational point groups of simple abelian varieties of dimension two over finite fields, Arch. Math. 63 (1994), 427-430.

[Xin96] Ch. Xing, On supersingular abelian varieties of dimension two over finite fields, Finite Fields Appl. 2 (1996), no. 4, 407-421.

Institute for Information Transmission Problems (RAS), Moscow, Russia

National Research University Higher School of Economics, Moscow, Russia

E-mail address: yuliakotel@gmail.com 